\title{
Spatiotemporal arrangement of organic matter and minerals in soils
}

\section{PROF. INGRID KOEGEL-KNABNER}

Chair of Soil Science, Chair of Soil Science, TU Muenchen

Presenting Author:koegel@wzw.tum.de

The amount and type of organic matter (among others alkyl C, polysaccharides) accumulated in soils is controlled, among other factors by intrinsic soil properties, specifically soil texture and the associated aggregate structures. Soil development leads to the formation of aggregated structures composed of a highly complex mixture of different mineral and organic constituents. The resulting soil type specific carbon sequestration can strongly be affected by soil management, varying greatly with the type and intensity of land use. The processes of formation and stabilization of organic matter through organo-mineral interactions in aggregated soil structures are controlled at the sub- $\mu \mathrm{m}$ scale. Understanding the binding of organic matter in these fine soil structures is thus key to elucidate the biogeochemical soil processes that are part of the carbon cycle as well as to evaluate the effects of soil management on the $\mathrm{C}$ cycle. I will discuss the state-of the-art, open questions for understanding these processes and how we can approach them by combining novel analytical techniques, such as nanoSIMS (secondary ion mass spectrometry) with innovative experiments. 